\title{
Efecto de Oxidantes y Emulsionantes sobre la Calidad del Pan elaborado con incorporación de Harina de Arvejas (Pisum sativum) inactivadas Enzimáticamente
}

\author{
María C. Alasino, Carlos A. Osella, María A. de la Torre y Hugo D. Sánchez* \\ Universidad Nacional del Litoral, Instituto de Tecnología de Alimentos, Facultad de Ingeniería \\ Química, $1^{\circ}$ de Mayo 3250-Santa Fe-Argentina (e-mail: hsanchez@fiq.unl.edu.ar) \\ * Autor a quien debe ser dirigida la correspondencia
}

Recibido Mar. 04, 2010; Aceptado May. 13, 2010; Versión Final recibida Jun.01, 2010

\section{Resumen}

Se estudió el efecto de oxidantes y emulsionantes sobre la calidad del pan elaborado con incorporación de harina de arvejas inactivadas. Se determinó volumen específico y se realizó evaluación sensorial. Se utilizó Análisis de Variancia (ANOVA) y Test de Rango Múltiple de Duncan. Se seleccionó el reemplazo de $10 \%$ de harina de trigo por harina de arvejas como situación de equilibrio entre mejora nutricional y deterioro tecnológico. La azodicarbonamida produjo resultados estadísticamente superiores a los obtenidos con ácido ascórbico. En emulsionantes el estearoillactilato de sodio produjo mejor comportamiento tecnológico que el monoglicérido de diacetiltartárico. Se concluye entonces, que con $10 \%$ de harina de arvejas se logra una mejora del 22,4\% en score químico.

Palabras claves: harina de arvejas, pan, oxidantes, emulsionantes, textura de miga

\section{Effect of Oxidants and Surfactants on bread quality made with Enzymatically inactivated Pea Flour (Pisum sativum)}

\begin{abstract}
The effect of oxidants and emulsifiers on the quality of bread made with the addition of inactivated pea flour was studied. Specific volume determination and sensory evaluation were carried out. Statistical tools were Analysis of Variance (ANOVA) and Duncan's Multiple Range Test. The replacement of $10 \%$ of wheat flour by pea flour was selected as equilibrium between nutritional improvement and technological deterioration. Azodicarbonamide gave better results than those obtained with ascorbic acid. Sodium stearoyl lactylate had better technological performance compared to the diacetyltartaric monoglyceride. It is concluded that with $10 \%$ pea flour the chemical score increased by $22.4 \%$ in chemical score.
\end{abstract}

Keywords: pea flour, bread, oxidants, surfactants, crumb texture 


\section{INTRODUCCIÓN}

Las proteínas provenientes de la arveja se complementan adecuadamente con las de harina de trigo para dar, desde el punto de vista nutricional, un mejor score químico (Lappé, 1991). No obstante se debe tener en cuenta el inconveniente que se presenta al utilizarla en panificación ya que la presencia de lipoxigenasa activa produce la aparición de olores y sabores desagradables. Por lo tanto esta lipoxigenasa debe inactivarse mediante la acción de un calentamiento moderado. Las condiciones de esta inactivación enzimática han sido ampliamente estudiadas por varios autores (Alasino et al., 2008; Drago et al., 2007; Garrote et al., 2001; Garrote et al., 1999).

El uso de agentes oxidantes en panificación generalmente produce una mejora de las propiedades de la masa y de la calidad del pan (Allen, 1999). Algunos autores como Miller y Hoseney (1999) sugieren que esas mejoras que suceden se deben atribuir a la generación de uniones disulfuro en la red del gluten. Uno de estos agentes oxidantes es el ácido ascórbico que actúa en su forma reducida modificando las propiedades reológicas de la masa (Nakamura y Kurata, 1997). El ácido ascórbico reacciona en presencia de la enzima ácido-ascórbicooxidasa de la harina, con pérdida de dos átomos de hidrógeno y formación de agua y ácido dehidroascórbico que es el agente oxidante que genera el aumento de elasticidad en la masa (Lu y Seib, 1998; Kaid et al.,1997; Every et al., 1996). Otro agente oxidante es la azodicarbonamida (ADA) que es de acción rápida pese a ser insoluble en agua y es recomendado en reemplazo de otros oxidantes como el bromato de potasio y el iodato de potasio. Su acción se identifica como oxidante de los grupos sulfidrilos (SH) a disulfuro (SS) generando un fortalecimiento de la masa al igual que el ácido ascórbico (Fitchett y Frazier, 1986).

Respecto de los emulsionantes, el monoglicérido de diacetiltartárico (DATEM) y el estearoillactilato de sodio (SSL) son muy utilizados en la industria de la panificación y ambos facilitan la interacción de los lípidos con las proteínas y el almidón. Las ventajas más reconocidas de su uso son el incremento del volumen de pan y el mejoramiento de la textura de la miga. También se les asigna la formación de complejos insolubles con la amilosa retardando así la capacidad de envejecimiento del pan (Beltrán-Orozco et al., 2007; Sluimer, 2005; Campbell et al., 2001; Ravi et al., 2000).

El objetivo de este trabajo fue estudiar el efecto del agregado de distintos niveles de oxidantes y emulsionantes sobre la calidad del pan de molde elaborado con la incorporación de $10 \%$ de harina de arvejas, inactivadas enzimáticamente, con la finalidad de obtener una mejora nutricional sin deterioro de las características del producto.

\section{MATERIALES Y MÉTODOS}

\section{Materias Primas}

Se utilizó harina de trigo comercial 000 procedente de la especie triticum vulgare, con humedad $12,6 \%$, gluten $27,0 \%$ y cenizas $0,62 \%$. Las propiedades físicas de la masa según el farinógrafo Do-Corder Brabender fueron: absorción de agua: 59,5 \%, tiempo de desarrollo: 2 min., estabilidad: 5,5 min. y ablandamiento 30 UB y según el alveógrafo Chopin: Energía W: 203 y Relación elasticidad/extensibilidad P/L: 1,7. Para estas determinaciones se utilizaron las técnicas estandares de AACC (1994).

Se trabajó con arveja deshidratada variedad denominada canadiense que fue enzimáticamente inactivada por inmersión en agua a $100^{\circ} \mathrm{C}$ durante $1,5 \mathrm{~min}$., enfriada inmediatamente por inmersión en agua a $20^{\circ} \mathrm{C}$ y luego secada por circulación forzada con aire a temperatura de $50^{\circ} \mathrm{C}$, siendo de $2,1 \%$ su actividad residual en lipoxigenasa. La harina obtenida por molienda en molino a martillos tuvo tamaño de partícula menor a $300 \mu \mathrm{m}$ y su composición química fue la siguiente: humedad $10,7 \%$, proteínas $21,4 \%$, materia grasa $2,1 \%$ y cenizas $3,4 \%$. 
Los aditivos utilizados fueron, como oxidantes (50-300 ppm) ácido ascórbico (AA) y azodicarbonamida (ADA) y como emulsionantes (0,7-2 \%) estearoíllactilato de sodio (SSL) y monoglicérido de diacetiltartárico (DATEM), todos ellos provistos por Nutring S.A., (Buenos Aires). Actividad de lipoxigenasa

La determinación se llevó a cabo mediante el Método Surrey modificado (Surrey, 1964) sobre la muestra de harina de arveja inactivada con tratamiento térmico de 1,5 min.

\section{Ensayo de panificación}

Los ingredientes utilizados en las formulaciones, con el reemplazo parcial de harina de trigo por harina de arvejas, fueron: $300 \mathrm{~g}$ de harina, $15 \mathrm{~g}$ de levadura, $6 \mathrm{~g}$ de $\mathrm{NaCl}, 18 \mathrm{~g}$ de azúcar, $9 \mathrm{~g}$ de oleomargarina y $6 \mathrm{~g}$ de leche en polvo. Se mezclaron durante un minuto en el farinógrafo DoCorder Brabender con amasadora de $300 \mathrm{~g}$ de capacidad. Luego se incorporó el agua con el aditivo a una temperatura tal que permitiera lograr una temperatura de masa de $24-26^{\circ} \mathrm{C}$, y se amasó durante $10 \mathrm{~min}$. a $60 \mathrm{rpm}$. En todos los casos se utilizó una hidratación de 59,5\%, que corresponde a la absorción de agua en farinógrafo para la harina de trigo. La fermentación se llevó a cabo en una cámara termostatizada a $27^{\circ} \mathrm{C}$ y con una humedad relativa del $75-80 \%$. El control de las etapas de fermentación se realizó con un medidor de empuje, que consiste en un cilindro de vidrio (75 mm de altura y $45 \mathrm{~mm}$ de diámetro) con un pistón que se eleva durante la fermentación. La primera fermentación concluye cuando se duplica el volumen de la masa, lo que ocurre en aproximadamente $40 \mathrm{~min}$. Luego se corta la masa en trozos de $200 \mathrm{~g}$, se preparan bollos, se dejan reposar durante $15 \mathrm{~min}$. y se arman las piezas, que luego se colocan en moldes engrasados y se llevan a segunda fermentación, que concluye cuando se cuadruplica el volumen inicial. Las medidas de los moldes metálicos son $5,5 \mathrm{~cm}$ de altura, $7 \mathrm{~cm} \times 17,5 \mathrm{~cm}$ de superficie en la base y $9 \mathrm{~cm} \times 18 \mathrm{~cm}$ en la parte superior. Las piezas son llevadas a cocción a $210^{\circ} \mathrm{C}$ durante 25 min. en horno eléctrico (Ojalvo S.A., Santa Fe, Argentina). Se retiran del horno, se dejan enfriar a temperatura ambiente durante 60 min.y se determina el volumen específico $(\mathrm{ml} / \mathrm{g})$ por el método de desplazamiento de semillas.

\section{Análisis Sensorial}

Esta evaluación sensorial de los panes fue llevada a cabo por un panel de tres expertos, cada uno con más de 20 años de experiencia en panificación, quienes asignaron puntaje de acuerdo al volumen específico y a las características organolépticas (Tosi et al., 2002). Los atributos evaluados y sus puntajes máximos son: Volumen: 15, Corteza: 15, Textura miga: 15, Color miga: 10, Estructura: 10, Aroma y Sabor: 35. De acuerdo al puntaje los panes fueron calificados de la siguiente manera: Excelente (90-100), muy bueno (80-89), bueno (70-79), aceptable (60-69), pobre (50-59), muy pobre (40-49), extremadamente pobre (30-39). A su vez, en los casos en que se trabajó con emulsionantes que presentan una conocida acción sobre la retrogradación del almidón, se llevó a cabo la evaluación de la textura de la miga en los dias 0, 1, 2, 3, 6 y 7 durante el envejecimiento de los panes, trabajando en el rango 0-100 sobre panes envasados en polipropileno y conservados a temperatura de $25^{\circ} \mathrm{C}$.

\section{Aspectos nutricionales}

Se evaluó la mejora nutricional con motivo de la incorporación de la harina de arvejas. A tal fin se efectuaron las determinaciones de proteínas por Kjeldahl y de lisina disponible según el método de Carpenter modificado por Booth (1971). El score químico se realizó por cálculo teniendo en cuenta los (mg aminoácidos/g proteína) / (mg aminoácidos/g proteína FAO para niños de 2-5 años de edad).

\section{Análisis Estadístico}

Todos los ensayos fueron realizados por triplicado para cada nivel de uso de aditivo y la herramienta estadística utilizada para comparación de valores medios, fue el ANOVA con el agregado del Test de Rango Múltiple de Duncan para establecer diferencias significativas (Montgomery, 1991). 


\section{RESULTADOS Y DISCUSIÓN}

Selección del nivel de suplementación con harina de arvejas

En Tabla 1 se muestran los resultados obtenidos con los distintos niveles de agregado de harina de arvejas a la masa para panificación.

Tabla 1:Resultados de la panificación con harina de arvejas sin aditivos (Letras diferentes indican que hay diferencia significativa a nivel de P menor o igual a 0.05)

\begin{tabular}{lccccc}
\hline & Testigo & \multicolumn{4}{c}{ Harina de arvejas (\%) } \\
\cline { 3 - 6 } & & 5 & 10 & 15 & 20 \\
\hline Vol. esp.(ml/g) & 4.29 & 4.08 & 3.79 & 3.55 & 3.10 \\
Puntaje total & $80^{\mathrm{a}}$ & $73^{\mathrm{b}}$ & $70^{\mathrm{c}}$ & $64^{\mathrm{d}}$ & $59^{\mathrm{e}}$ \\
Score químico & 52 & 65 & 74 & 86 & - \\
Lisina disponible & 2.28 & 2.87 & 3.25 & 3.77 & - \\
(g/100g prot.) & & & & &
\end{tabular}

Estos ensayos previos nos permitieron seleccionar el nivel de $10 \%$ de suplementación de harina de trigo con harina de arvejas inactivadas para desarrollar el presente trabajo en el que se estudió la incorporación de oxidantes o emulsionantes. Esta selección se basó en tener en cuenta la buena calificación por parte del panel (puntaje total=70) y la importante mejora nutricional lograda (score químico=74). Este hecho es coincidente con las recomendaciones de Repetsky y Klein (1981) quienes consideran que el $10 \%$ de reemplazo es el máximo admisible en un producto panificado.

\section{Efecto de aditivos oxidantes}

En Tabla 2 se presentan los valores obtenidos de volumen específico y puntaje total para los panes elaborados con $10 \%$ de harina de arvejas con la incorporación de distintos niveles de aditivos oxidantes. En los valores de volumen específico no existe mejora significativa, respecto al testigo con $10 \%$ de harina de arvejas y sin aditivos, cuyo valor era $3.79 \mathrm{ml} / \mathrm{g}$, en cambio se aprecia un aumento en las características sensoriales que aportan al puntaje total, ya que superan el valor 70 que pertenece a la referencia. El oxidante ADA presenta un mejor comportamiento que el ácido ascórbico como se aprecia en las figuras 1 y 2 y se destaca esa diferencia estadísticamente significativa a través de los valores promedio de Tabla 1 tanto para volumen específico como para puntaje total. En volumen específico el valor promedio con ADA supera en un $25 \%$ y en puntaje total en un $3 \%$ a los respectivos valores promedios obtenidos con AA. Estas diferencias estadísticamente significativas nos permiten seleccionar al ADA para futuros trabajos en la elaboración de panes con harina de arvejas en donde se combine este aditivo con algun agente emulsionante.

Tabla 2: Efecto de oxidantes en pan con 10\% de harina de arvejas

(Letras diferentes indican que hay diferencia significativa a nivel de P menor o igual a 0.05 )

\begin{tabular}{|c|c|c|c|c|}
\hline \multirow[b]{2}{*}{ ppm } & \multicolumn{2}{|c|}{ Vol. Esp.(ml/g) } & \multicolumn{2}{|c|}{ Puntaje total (máx.100) } \\
\hline & ADA & AA & ADA & AA \\
\hline Control & \multicolumn{2}{|c|}{$3.79^{b}$} & \multicolumn{2}{|c|}{$70^{g}$} \\
\hline 50 & $3.94^{\mathrm{a}}$ & $3.10^{\mathrm{de}}$ & $75.8^{\mathrm{a}}$ & $72.0^{\text {ef }}$ \\
\hline 100 & $3.53^{c}$ & $2.99^{\mathrm{ef}}$ & $73.8^{\mathrm{bcd}}$ & $72.5^{\mathrm{e}}$ \\
\hline 150 & $3.76^{b}$ & $2.96^{\mathrm{ef}}$ & $74.4^{b}$ & $72.9^{\mathrm{de}}$ \\
\hline 200 & $3.41^{\mathrm{c}}$ & $2.85^{\mathrm{fg}}$ & $74.1^{\mathrm{bc}}$ & $72.3^{\mathrm{e}}$ \\
\hline 250 & $3.26^{\mathrm{d}}$ & $2.53^{\mathrm{h}}$ & $73.8^{\mathrm{bcd}}$ & $71.1^{f}$ \\
\hline 300 & $3.18^{d}$ & $2.74^{\mathrm{g}}$ & $73.5^{\text {cde }}$ & $71.2^{f}$ \\
\hline Promedio & $3.51^{\mathrm{c}}$ & $2.86^{\mathrm{fg}}$ & $74.2^{\mathrm{bc}}$ & $72.0^{\mathrm{ef}}$ \\
\hline
\end{tabular}


Estos resultados muestran coincidencia con Joye et al. (2009) quienes establecen que las deficiencias encontradas en la panificación pueden ser superadas por el agregado de componentes exógenos que mejoran la funcionalidad de las proteínas del gluten durante el proceso. Estos autores manifiestan además que ciertos aditivos como la azodicarbonamida promueven la formación de enlaces covalentes entre las proteínas del gluten provocando un mejoramiento de la calidad del pan.

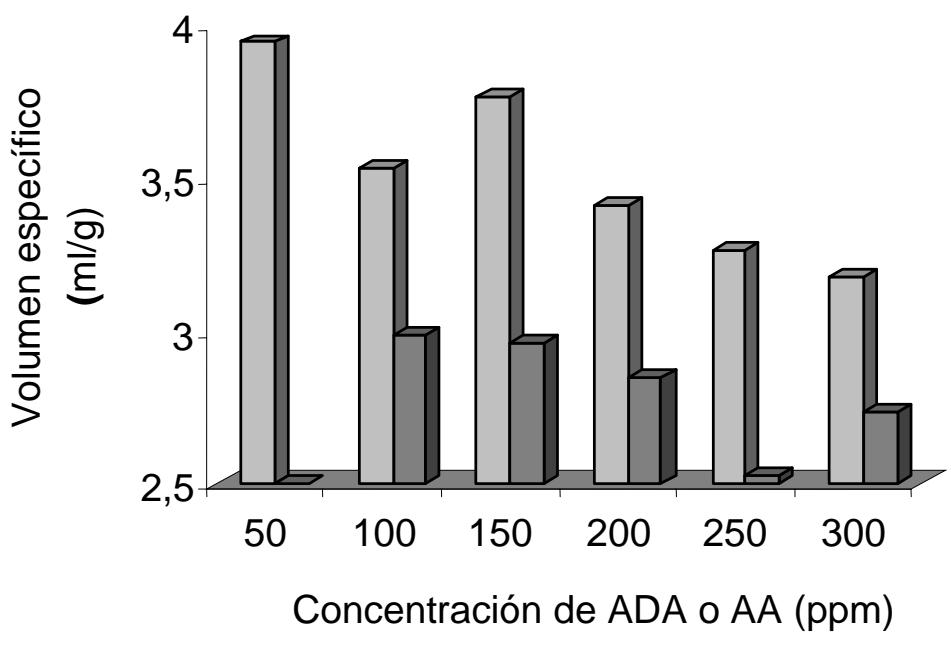

ADA

$A A$

Fig. 1: Efecto de azodicarbonamida (ADA) y de ácido ascórbico (AA) sobre el volumen específico del pan con $10 \%$ de harina de arvejas

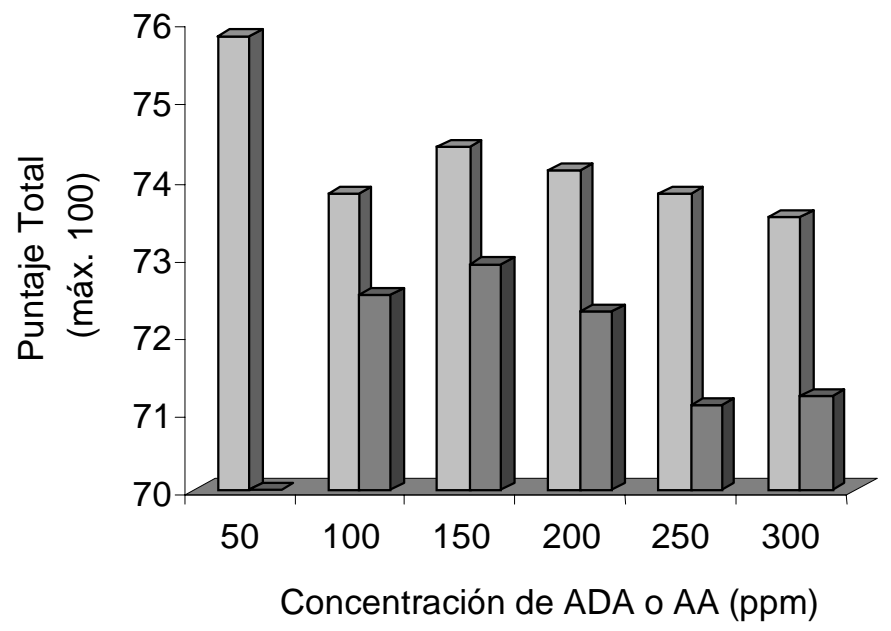

ADA

AA

Fig. 2: Efecto de azodicarbonamida (ADA) y de ácido ascórbico (AA) sobre el puntaje total del pan con $10 \%$ de harina de arvejas

Efecto de aditivos emulsionantes

En Tabla 3 se presentan los valores obtenidos de volumen específico y puntaje total para los panes elaborados con $10 \%$ de harina de arvejas con la incorporación de distintos niveles de aditivos emulsionantes. Los valores promedios muestran con significación estadística que el 
emulsionante SSL produjo los efectos más destacados sobre el volumen específico y sobre las características sensoriales. Además se puede apreciar en las figuras 3 y 4 la evolución de este mejoramiento del volumen específico y de la evaluación sensorial a través de dicho puntaje total. Este aditivo produjo un positivo impacto tecnológico no sólo al tener un mejor comportamiento que el DATEM en esta circunstancia, sino que superó en aproximadamente un $20 \%$ los valores de volumen específico y puntaje total del pan obtenido con $10 \%$ de harina de arvejas sin aditivos. El agregado de $1,5 \%$ de SSL resultó ser el valor recomendado para panificar con un nivel de sustitución del $10 \%$ de harina de arvejas (Fig. 5). En lo relativo al estudio del envejecimiento de dichos panes, en la figura 6 se puede apreciar la eficiente acción lograda sobre la textura de la miga con la utilización del SSL. Las ventajas apreciadas sobre la textura de la miga son compartidas por Eliasson (1983) quien establece que el agregado de SSL retarda la gelatinización del almidón, lo que el autor verifica utilizando la calorimetría diferencial de barrido (DSC) donde en la transición obtiene un descenso del valor entálpico pero a mayor temperatura. Pequeñas cantidades de SSL además de retardar la gelatinización del almidón, retardan su cristalización, con lo que también se retarda su envejecimiento. En cambio, con elevados niveles de SSL se forman complejos con la amilosa que lleva a retardar la gelatinización sin tener un efecto sobre la cristalización del almidón o sea sobre el envejecimiento del pan.

Tabla 3: Efecto de emulsionantes en pan con $10 \%$ de harina de arvejas (Letras diferentes indican que hay diferencia significativa a nivel de $\mathrm{P}$ menor o igual 0.05 )

\begin{tabular}{cccccc}
\hline \multirow{2}{*}{$\%$} & \multicolumn{2}{c}{ Vol. Esp.(ml/g) } & \multicolumn{2}{c}{ Puntaje total } \\
\cline { 2 - 6 } & DATEM & SSL & DATEM & SSL \\
\hline Control & & $3.79^{\mathrm{c}}$ & & $74.5^{\mathrm{c}}$ & $70^{\mathrm{e}}$ \\
0.7 & $3.44^{\mathrm{d}}$ & & $3.84^{\mathrm{c}}$ & $75.0^{\mathrm{c}}$ \\
1.0 & $3.48^{\mathrm{d}}$ & $4.18^{\mathrm{b}}$ & $74.5^{\mathrm{c}}$ & $78.5^{\mathrm{b}}$ \\
1.5 & $3.38^{\mathrm{d}}$ & $4.57^{\mathrm{a}}$ & $69.5^{\mathrm{e}}$ & $81.5^{\mathrm{a}}$ \\
2.0 & -- & $4.49^{\mathrm{a}}$ & -- & $79.0^{\mathrm{b}}$ \\
\hline Promedio & $3.43^{\mathrm{d}}$ & $4.27^{\mathrm{b}}$ & $72.8^{\mathrm{d}}$ & $78.5^{\mathrm{b}}$ \\
\hline
\end{tabular}

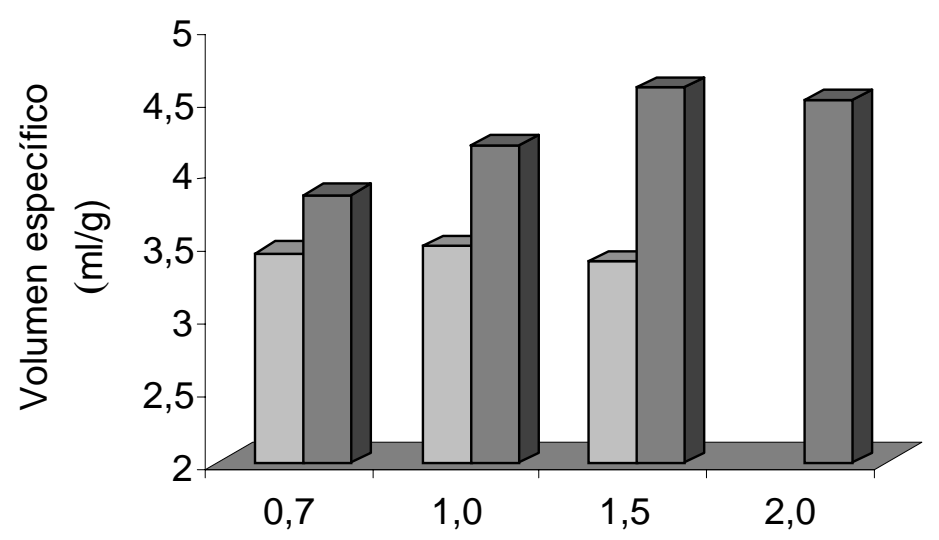

Concentración de DATEM o SSL (\%)

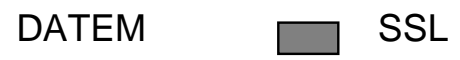

Fig. 3: Efecto de monoglicérido de diacetiltartárico (DATEM) y de estearoíllactilato de sodio (SSL) sobre el volumen específico del pan con $10 \%$ de harina de arvejas 


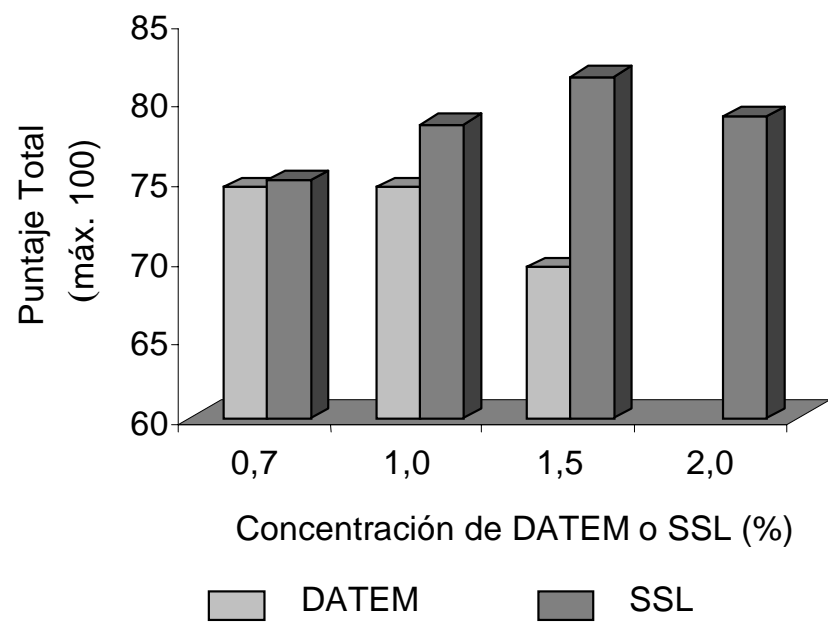

Fig. 4: Efecto de monoglicérido de diacetiltartárico (DATEM) y de estearoíllactilato de sodio (SSL) sobre el puntaje total del pan con $10 \%$ de harina de arvejas

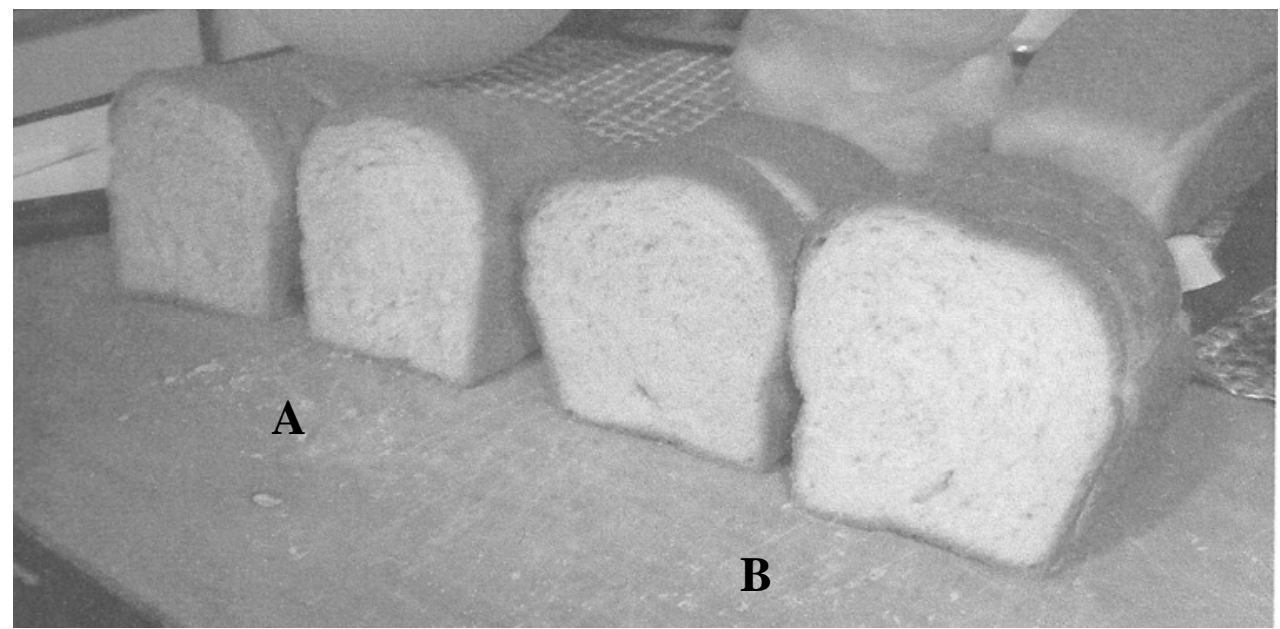

Fig. 5: Panes elaborados con harina de arvejas al $10 \%$ y con SSL al $0,7 \%(\mathrm{~A})$ y al $1,5 \%(\mathrm{~B})$

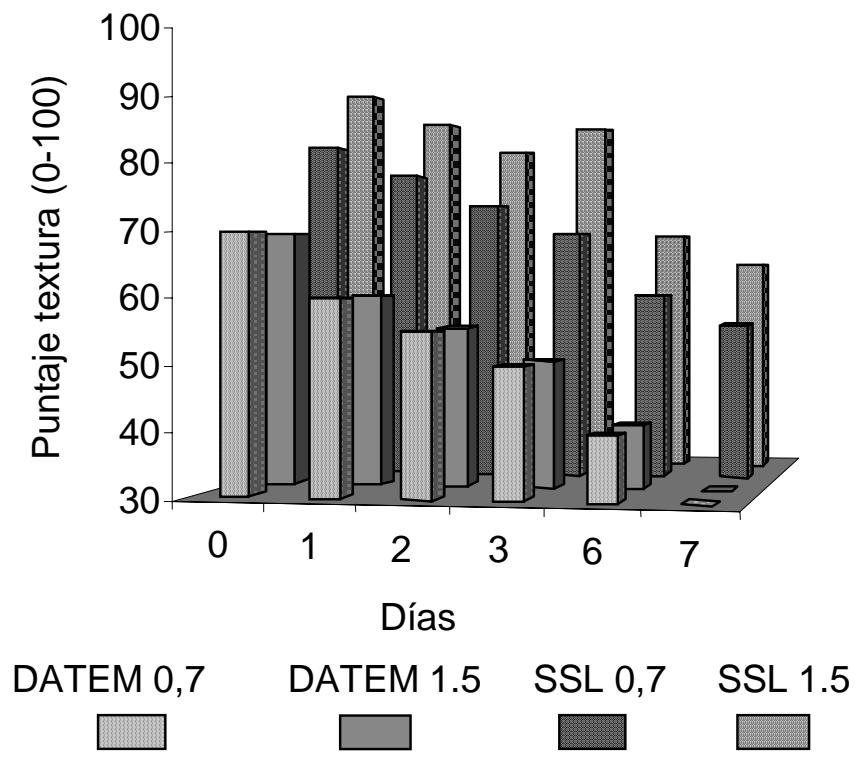

Fig. 6: Efecto, durante el almacenamiento, de monoglicérido de diacetiltartárico (DATEM) y de estearoíllactilato de sodio (SSL) sobre la textura de la miga del pan con $10 \%$ de harina de arvejas. 


\section{CONCLUSIONES}

Respecto a los oxidantes y emulsionantes estudiados se destaca que el ADA fue más eficiente que el AA y que el SSL tuvo un mejor comportamiento que el DATEM, llegando en este último caso a superarlo en un $23 \%$ en volumen específico. Este beneficio logrado con ADA y SSL en el proceso de panificación deberá ser estudiado en un futuro trabajo de investigación a través de su uso combinado, optimizando sus proporciones.

Adicionalmente se puede agregar que desde el punto de vista nutricional se logra un producto mejorado en su valor biológico al reemplazar el $10 \%$ de harina de trigo por harina de arvejas inactivadas enzimáticamente. Se consigue un aumento en proteínas totales del 10,7\%, en lisina disponible del $58 \%$ y en score químico del $22,4 \%$, siempre con referencia a los panes que contienen harina de trigo solamente.

\section{REFERENCIAS}

American Association of Cereal Chemists. Approved Methods of the AACC. Methods 38-10, 0801, 54-21, 54-30, The Association: St. Paul, MN. (1994).

Alasino, M.C. y otros 5 autores, Panificación con Harina de Arvejas (Pisum Sativum) Previamente Sometidas a Inactivación Enzimática. Archivos Latinoamericanos de Nutrición, 58(4), 397- 402 (2008).

Allen, W., Alternative Oxidants as Dough Conditioners, Cereal Foods World, 44, 642-649 (1999).

Beltrán-Orozco, M.C.; J.H. Rendón Meza y T. Gallardo-Velázquez, Cinética de las Características Físicas de Mantecadas Bajas en Grasa Almacenada en dos tipos de Material de Empaque durante su vida de Anaquel, Información Tecnológica, 18 (3), 13-22 (2007).

Booth,V.H., Problems in the Determination of FDNB-Available Lysine, Journal of Food Science and Agriculture, 22 (12), 658-666 (1971).

Campbell, G.M., R. Herrero-Sánchez, R. Payo-Rodriguez y M.L. Merchan, Measurement of Dynamic Dough Density and Effect of Surfactants and Flour Type on Aeration during Mixing and Gas Retention during Proofing, Cereal Chemistry, 78, 272-277 (2001).

Drago, S.R., R.J. González, L. Chel-Guerrero y M.E. Valencia, Evaluación de la Disponibilidad de Minerales en Harinas de Frijol y en Mezclas de Maíz/Frijol Extrudidas, Información Tecnológica, 18 (1), 41-46 (2007).

Eliasson, A.C., Differential Scanning Calorimetry Studies on Wheat Starch-gluten Mixtures: II. Effect of Gluten and Sodium Stearoyl Lactylate on Starch Crystallization during Aging of Wheat Starch Gels, Journal of Cereal Science, 1 (3), 207-213 (1983).

Every, D., M.J. Gilpin y N.J. Larsen, Ascorbate Oxidase Levels in Wheat and Relationship to Baking Quality, Journal of Cereal Science, 23, 145-151 (1996).

Fitchett, C. y P. Frazier, Action of Oxidants and other Improvers. In Chemistry and Physics of Baking. Edited by J. Blanshard; P.Frazier; T. Galliard, 179-198. The Royal Society of Chemistry. Burlington House. London UK. (1986).

Garrote, R.L., E.R. Silva y R.A. Bertone, Kinetics Parameters for Thermal Inactivation of Cut Green Beans Lipoxygenase Calculated Using Unsteady-state Methods, International Journal of Food Science and Technology, 36, 377-385 (2001).

Garrote, R.L., E.R. Silva, R.A. Bertone y R.D. Roa, Predicting the End Point of a Blanching Process, Lebensmittel Wissenschaft und Technologie 37, 309-315 (2004). 
Joye, I.J., B. Lagrain y J.A. Delcour, Use of Chemical Redox Agents and Exogenous Enzymes to Modify the Protein Network during Bread Making, Journal of Cereal Science, 50 (1), 11-21 (2009).

Kaid, N., L. Rakotozafy, J. Potus y J. Nicolas, Studies on the Glutathione-dehidroascorbate Oxidoreductase from Wheat Flour, Cereal Chemistry, 74, 606-610 (1997).

Lappé, F.M., Diet for a Small Planet. 20 th Aniv. Edit. Ballantine Books. New York. (1991)

Lu, X. Y P. Seib, Assay of Dehidroascorbic Acid in Bread and Dough added as a Crystaline Dimer, Cereal Chemistry, 75, 200-205 (1998).

Miller, K.A. y R.C. Hoseney, Effect of Oxidation on the Dynamic Rheological Properties of Wheat Flour-water Doughs, Cereal Chemistry, 76, 100-104 (1999).

Montgomery D., Diseño y Análisis de Experimentos, $3^{a}$ edición 589-590. Grupo Editorial Iberoamericana. Mexico (1991).

Nakamura, M. y T. Kurata, Effect of L-ascorbic Acid on the Rheological Properties of Wheat Flourwater Dough, Cereal Chemistry, 74, 647-650 (1997).

Ravi, R., R. Sai-Manohar y P. Haridas Rao, Influence of Additives on the Rheological Characteristics and Baking Quality of Wheat Flours, European Food Research and Technology, 210, 202-208 (2000).

Repetsky, J.A. y B.P. Klein, Partial Replacement of Wheat Flour with Yellow Field Pea Flour in White Pan Bread, Journal of Food Science, 47, 326-327 (1981).

Sluimer, P., Principles of Breadmaking. Functionality of Raw Materials and Process Steps, $1^{\text {a }}$ edición, 53-58. American Association of Cereal Chemists Inc. St. Paul, Minnesota, USA (2005).

Surrey, K., Spectrophotometric Method for Determination of Lipoxidase Activity, Plant Physiology, 39, 65-70 (1964).

Tosi, E.A. y otros 5 autores, Whole and Deffated Hyperproteic Amaranth Flours Tested as Wheat Flour Supplementation in Mold Breads, Lebensmittel Wissenschaft und Technologie, 35, 472-475 (2002). 
